\title{
Measuring complex for examination the combustion products' composition and the soot particulate sol microstructure
}

\author{
N I Moskalenko, I R Dodov, M S Khamidullina, Ya S Safiullina \\ Kazan State Power Engineering University, Russia, 420066, Kazan, Krasnoselskaya 51
}

\begin{abstract}
A measuring complex of spectral instrumentation for examination of the ingredient composition, optical characteristics and microstructure of the dispersed phase of the combustion products of energy fuels and atmospheric anthropogenic emissions is discussed. The measuring complex includes a multi-pass cell with external and internal electric heating, which makes it possible to change the temperature of the radiation propagation medium in a multi-pass cell in the temperature range of $300-800 \mathrm{~K}$, in the pressure range of $1 * 10^{2} \leq \mathrm{P} \leq 3 * 10^{6} \mathrm{~Pa}$ with optical path $\mathrm{L}=2,4,6, \ldots 30 \mathrm{~m}$. Registration of spectra is performed by an upgraded spectrometer IKS-31 with interchangeable sources and radiation receivers, interchangeable diffraction gratings and cut-off light filters, interchangeable quartz windows, CaF2, LiF, MgF2, IKS-25, KRS-5, ensuring their operation in the spectral region of $0.2-100 \mu \mathrm{m}$ with the spectral resolution limit $\Delta v=$ $0.2 \mathrm{~cm}^{-1}$. The measuring complex has a complete metrological assurance. The obtained data on the spectral dependences of the effective cross sections for absorption, scattering, and attenuation of radiation are used to restore the microstructure of the dispersed phase of the combustion products. The atmospheric emissions of combustion products of wood, industrial energy, air carriers, decomposition products of asbestos-cement slabs during their calcinations were analyzed.
\end{abstract}

\section{Introduction}

Natural and anthropogenic emissions into the atmosphere during the operation of power plants at the power engineering enterprises, transport, and combustion products of vegetation already affect the radiation heat exchange in the atmosphere, the weather and the climate of the Earth, the temporal trends of which are already apparent now [1-2]. Information on the composition of the combustion products of energy fuels is also required for calculation of radiative heat exchange in the combustion chambers of power engineering plants. Below we consider a spectral measuring complex for examination of composition of anthropogenic emissions into the atmosphere and combustion products of energy fuels.

\section{Measuring complex for examination of composition of anthropogenic emissions into the atmosphere and combustion products of energy fuels}

The spectrometric analytical laboratory is a heated spectrophotometric complex, which uses a multi-pass working chamber (MPC) with internal and external heating as a working chamber. The heating system allows one to change the temperature of the working medium in the temperature range of 300-900 K. The evacuated monochromator and illuminator enable analysis of qualitative and quantitative composition of the combustion products of the selected samples. The need to develop a heated working chamber arose due to the need to measure the multicomponent composition of combustion products, which include volatile and nonvolatile components in normal atmospheric conditions with condensation temperatures ranging from 30 to $150^{\circ} \mathrm{C}$, as well as the ash fraction of the dispersed phase. Under normal atmospheric conditions, many components are captured by ash particles or dissolved in water droplets. Thus, the measurement results of widely used technologies become unrepresentative and do not show the actual composition of the combustion products.

The use of a working chamber with a multi-path scheme for the passage of radiation allows one to change the length of the optical path (measurement sensitivity) during the measurement process. If necessary, the pressure range in the working chamber can be expanded by diluting the sample with an inert optically inactive gas. High spectral resolution allows identifying the components of the combustion products by the fine structure of the absorption spectra. MPC has both internal and external heating. When using external heating, the internal cavity of the cuvette is heated to a temperature of $120-150{ }^{\circ} \mathrm{C}$, and internal electric heating allows the gas temperature to rise to $800 \mathrm{~K}$. The internal furnace is mounted between the mirrors of the MPC white optical bench in a cylindrical heat-insulating casing made of asbestos-cement calcined at temperature $\mathrm{T}=1000 \mathrm{~K}$. Reflective diaphragms are placed at the 
ends of furnace. The temperature along the optical path of the radiation beam is controlled by five thermocouples with an error of $\pm 3{ }^{\circ} \mathrm{C}$. After equilibrium is established, the temperature difference at the furnace ends with respect to its central part at $\mathrm{T}=300 \mathrm{C}$ is $\sim 20^{\circ} \mathrm{C}$. The electric furnace and multi-way cell are designed in such a way that at pressures $\mathrm{P}<0.1 \mathrm{MPa}$ and the temperature of the gas mixture $\mathrm{T} \leq 800 \mathrm{~K}$, the temperature of the windows of the cell is close to room temperature, and the body temperature does not exceed $80{ }^{\circ} \mathrm{C}$. The body of the cuvette is a prefabricated structure made of stainless steel. All metal assemblies are connected using hermetic seals made of high-temperature rubber plates.

The degree of heating is regulated by voltage applied to the electric furnace from the AOMN-40-220 autotransformer. Pumping out the modules of the measuring complex and launching the medium under study is carried out by vacuum-metering system, which is connected to the cuvette using heated stainless steel pipelines. The samples are heated to temperature $\mathrm{T}=150$ ${ }^{\circ} \mathrm{C}$ before their introduction in the MPC. The sample is introduced into a vacuum chamber preheated to a temperature $\mathrm{T}=150{ }^{\circ} \mathrm{C}$ and in an evacuated working chamber. To study the impact of relative humidity $f$ on the optical characteristics of anthropogenic sols in the MPC, a steam generator of distilled water of natural isotopic composition is used. Relative humidity $f$ was determined by the temperature and partial pressure of water vapor in the MPC.

The optical setup is shown in Fig. 1. Illuminator I and monochromator III with receiving chamber IV of the upgraded IKS-31 spectrometer are spaced relative to each other. Radiation modulated with a frequency $\mathrm{f}=$ $12.5 \mathrm{~Hz}$ passes through a heated cell II and mirrors 10 and 11 is focused in the plane of the entrance slit 23 of the monochromator, which is located at the focus of the spherical mirror 15 (I). By mirrors 13 and 15 (I), the radiation is directed to the diffraction grating 14 . After diffraction, the beam of parallel rays is collected by a spherical mirror 15 (II) and is guided by a rotating mirror 16 into the receiving chamber IV and further by a mirror 18 - to the receiving platform of the receiver $\mathrm{V}$ For cutting the spectra of the overlapping orders of the diffraction grating after the exit slit of the monochromator, a block of replaceable cut-off filters 17 is installed. A graphic form was used to record the radiation attenuation spectra. The spectral range of operation of the IKS-31 spectrometer was expanded due to the additional configuration of diffraction gratings, interchangeable radiation detectors, cut-off and bandpass light filters, ensuring its operation in the spectral range of 0.2-100 Im with a spectral resolution $\Delta v=0.2$ $\mathrm{cm}^{-1}$. The spectral calibration of the upgraded IKS-31 spectrometer was performed according to the position of the centers of the spectral lines $\mathrm{SO}_{2}, \mathrm{NO}_{2}$, water vapor $\mathrm{H}_{2} \mathrm{O}, \mathrm{CO}, \mathrm{HCl}$. The length of the optical path in the MPC is 2, 4, 6, .., $30 \mathrm{~m}$.

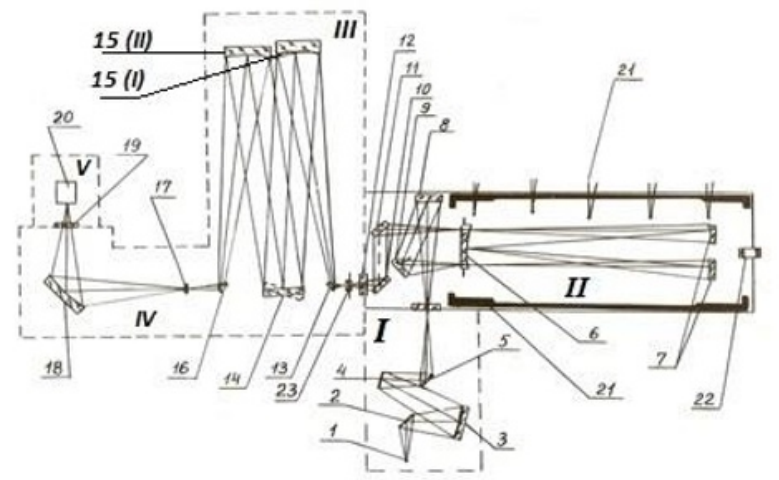

Fig. 1. Optical setup: $2,3,4,5$ - mirrors of the optical prefix of the illuminator I with a replaceable radiation source 1 (globar or TRSh-2580 ribbon lamp for the UV and visible spectral regions); 8,9,10,11 - mirrors of the optical set-top box for matching the MPC II with the illuminator I; 12, 19- protective windows of the modules of the camera of the spectrometer IKS-31; 6,7 - front and rear spherical mirrors of the optical bench of White MPC-1; 21 - thermocouples; 22 - eyepiece to observe the number of multiple passage of radiation in the MPC.

The radiation beam, after repeated passage between the front and rear mirrors, is focused in the plane of the output diaphragm of White's optical system (White). Input and output diaphragms of MPC are interchangeable with a size of slits $6 \times 15 ; 2 \times 15 ; 1 \times 15$ $\mathrm{mm}$. Replaceable windows MPC and spectrometer are made of quartz, $\mathrm{CaF}_{2}, \mathrm{BaF}_{2}, \mathrm{LiF}, \mathrm{MgF}_{2}, \mathrm{KRS}-5$, IKS-25. When measuring in the UV and visible spectral ranges, photomultipliers FEU-39A and FEU-62 are used. The pressure and vacuum in the MPC are measured with arrow gauges and model 1227 vacuum gauges, a mercury gauge and a vacuum gauge VDG-1.

The analytical laboratory was tested when measuring the composition of the combustion products of automotive fuel, pine timber combustion products in model fire tests in combustion chambers [3-5], in fullscale fire tests of a fragment of the PLASTBAU building [6]. In the latter case, the measurements were performed during the entire fire period with full control of the temperature field reconstructed from thermocouple readings, at sampling points and in the vicinity of the firing experiment. The maximum temperature of the combustion products of pine timber was $T_{\max }=(1500 \pm$ 50) K. Monitoring of the composition of the combustion products was carried out by fine-structure spectrometry method from the moment of ignition to the end of fire. Based on the data of spectrometric measurements, 34 optically active ingredients have been found and identified, most of which are volatile and heavy nonvolatile hydrocarbons.

\section{The study of the optical characteristics of anthropogenic sols}

The spectral dependence of the attenuation coefficients of the radiation by the dispersed phase of the combustion products makes it possible to divide it into fractions 
corresponding to the mechanisms of their generation: the mineral sol component of the fuel particles; fraction caused by bursting of films during the degassing of burning fuel particles; fraction generated by the mechanism of ionic nucleation of soot particles from the gas phase of the combustion products.

Theoretical models and experimental results of the processes of soot formation and burning out during the combustion of hydrocarbon fuels are analyzed in [7-8].

Our experimental studies have shown that the sol generated in the furnace space is hygroscopic, which indicates the presence of soluble components in the soot particles that make up various fuels from 10 to $20 \%$ of the mass of soot in the particles. The minimum value of $10 \%$ mass concentration of soluble components corresponds to the soot sols when burning gas fuel. The maximum value of $20 \%$ of the mass concentration of soluble components corresponds to the soot sols when burning wood. Measurements of the density of the carbon black sol showed that it depends on the combustion mode and varies within the range of $\rho \in\{1.9$, 2.4 $\mathrm{g} / \mathrm{cm}^{3}$. In accordance with the indicated composition of the sol particles, the optical characteristics of model microstructures of soot particles having a spherical shape were calculated for modified gamma distributions with modal particle radii from $1.6 \cdot 10^{-3}$ to $2.25 \mu \mathrm{m}$. To determine the microstructure of the carbon black sol from the data of spectral optical measurements, an electronic database of the optical characteristics of the carbon black sol was used, obtained using spectral values of the refractive index and carbon black absorption [10].

The microstructure of atmospheric emissions of anthropogenic soot black sol depends on many factors: the elemental composition of the fuel, the residence time of the combustion products in the flame zone, the electrical properties of the combustion products that determine the rate of coagulation and the agglomeration of the primary carbon black sol. The mechanisms of carbon black sol formation were investigated using flame measuring complexes operating in the spectrometry and spectroradiometry modes described in [3]. Homogeneous coagulation of sol particles is described by a simple ratio

$$
\frac{r}{r_{0}}=\left(1+\frac{1}{2} k_{\Delta_{0}} \ln \left\{\frac{(1+\alpha t)}{\alpha}\right\}\right)^{\frac{1}{3}},
$$

Where $k$ is the Brownian coagulation coefficient, $n_{0}$ is the number of particles per unit volume, $\alpha^{-1}$ is the time during which the radius of the particles doubles.

For heterogeneous multicomponent coagulation of particles, the distribution of the number of particles $f[r(t)]$ is determined by the ratio

$$
\begin{aligned}
& \frac{f[r(t)]}{f_{0}(r)}=\sum_{i}\left[1+\frac{1}{2} k_{i} n_{0 i} \ln \left\{\frac{1+\alpha_{i} t}{\alpha_{i}}\right\}\right]^{\frac{1}{3}}+ \\
& +\sum_{i \neq k}\left[1+\frac{1}{2} k_{i k}\left(n_{0 i} n_{0 k}\right)^{\frac{1}{2}} \ln \left\{\frac{1+\alpha_{i k} t}{\alpha_{i k}}\right\}\right]^{\frac{1}{3}},
\end{aligned}
$$

where $f_{0}(r)=\sum_{i} n_{i}, n_{i}$ are the number of particles with a radius $r_{i}$ per unit volume; $f[r(t)]$ is the time dependence of the distribution of the number of particles in size; $i$ is the fraction number; $k_{i}$ is the Brownian coagulation coefficient for the component $i ; k_{i_{k}}$ is the coefficient of Brownian interaction of particles of different fractions $i, k$.

When performing calculations $f[r(t)]$ it is possible to use an iterative procedure in time calculations in increments $\Delta t$. Experiments show that the coagulation of sol particles is strongly influenced by the electrical properties of the particles.

When burning gas fuel, a sol with a modal radius $r_{m}=0.003 \mathrm{Im}$ is observed, which is more finely dispersed when sol, obtained when burning methane. With an increase in the $\mathrm{C} / \mathrm{H}$ ratio of hydrocarbon molecules, the modal radius $r_{m}$ of soot particles increases.

Determining the microstructure of the carbon black sol is an inverse problem and for its solution requires a priori information on the optical (radiation) characteristics of the carbon black sol of various microstructural formations that cover the entire range of variations in the spectral dependences of the radiation attenuation coefficient. In this case, the reference wavelengths (measurement channels) are selected in the transparency windows of the gas phase of the combustion products with the minimum contribution of radiation absorption to the recorded emission attenuation spectra and include the wavelengths in the UV, visible and IR spectral ranges. Optimization of solution to the problem of restoring the microstructure of a sol from the radiation attenuation spectra is performed from the condition:

$$
\sum_{i} \frac{\left|\Delta \sigma_{\alpha \lambda i}\right|}{\sigma_{\alpha \lambda i}}=\min
$$

where $\sigma_{\alpha \lambda i}$ is the deviation in the spectral dependence of the attenuation coefficient of the reconstructed spectrum $\sigma_{\alpha \lambda i}$ from the measured one, $i$ is the number of the measurement channel. Table №1 shows the models of carbon black sol microstructure, which are used to restore the actual microstructures of the dispersed phase of the combustion products. For the optimal choice of the zero approximation, an additional database of zero approximations is created. The choice of the zero approximation is determined by the ratio (3). The 
Table 1. Soot microstructure models.

\begin{tabular}{|l|l|l|l|l|l|l|l|l|l|}
\hline № & $a$ & $b$ & $\gamma$ & $r_{m}$, m & № & $a$ & $b$ & $\gamma$ & $r_{m}, \mu m$ \\
\hline 1 & 1 & 50 & 0.5 & $1.6 \cdot 10^{-3}$ & 6 & 1.5 & 6 & 1 & $2.5 \cdot 10^{-1}$ \\
\hline 2 & 0.2 & 6 & 0.5 & $4.4 \cdot 10^{-3}$ & 7 & 0.1 & 0.5 & 2 & 0.3 \\
\hline 3 & 1 & 9 & 0.5 & $4.9 \cdot 10^{-2}$ & 8 & 1 & 1 & 2 & 0.3 \\
\hline 4 & 1 & 7.5 & 0.5 & $9 \cdot 10^{-2}$ & 9 & 3 & 6 & 0.5 & 1 \\
\hline 5 & 2 & 12 & 7.5 & $8.9 \cdot 10^{-2}$ & 10 & 3 & 4 & 0.5 & 2.25 \\
\hline
\end{tabular}

parameters of models $a, b, \gamma, r_{m}$ of the microstructure of the black sol of modified gamma distributions $f(r)=A r^{a} \exp \left(-\gamma r^{b}\right), \quad r_{m}$ the modal distribution radius are presented in Table 1.

The procedure for determining the microstructure of the particulate sol is performed in the following order. The microstructure of the database of zero approximations and gamma distributions are set from Table 1 for values $\tau_{0}=\tau(\lambda=0.55 \mu \mathrm{m})=1$. The zero approximation and the effective radiation attenuation cross sections for the selected channels (wavelengths) are chosen. The values $\sum_{i} \frac{\left|\Delta \sigma_{\alpha \lambda i}\right|}{\sigma_{\alpha \lambda i}}$ are calculated depending on the optical characteristics.

Thickness $\tau_{i}$ of the selected microstructure $N_{l}(r)$ of table 1 is determined by the value $\tau_{\min }$, for which $\sum_{i} \frac{\left|\Delta \sigma_{\alpha \lambda i}\right|}{\sigma_{\alpha \lambda i}}=\min$. Next, the microstructure $N(r)$ is calculated by the ratio

$$
N(r)=N_{0}(r)\left[1-\tau_{\min }\right]+N_{1}(r) \tau_{\min },
$$

The obtained value of $N(r)$ is assigned a zero approximation and the procedure is repeated for the next microstructure sample from Table 1. The procedure for determining $N(r)$ is completed after a complete enumeration of microstructures of gamma distributions from Table 1. The error in calculating the optical density $\frac{\Delta \tau_{i}}{\tau_{i}}$ on restored microstructures makes $3 \div 4 \%$.

\section{Conclusions}

Fig. 3 shows the size-distribution function of the number of particles $f(r)$ for methane-air $A$, propane-air $B$, acetylene-air flames $C$, emissions of combustion products of the gasoline engine 1 and the combustion products of pine timber 2. Normalization $f(r)$ is performed by the ratio $\int f(r) d r=1$. Combustion of gas fuel is diffusive for an optimal combustion mode $[11,12]$ with an excess air coefficient $\alpha=1.03$ from a multi-row burner [3]. Flame zone height is $0.3 \mathrm{~m}$, flow rate of combustion products is $15 \mathrm{~m} / \mathrm{s}$.

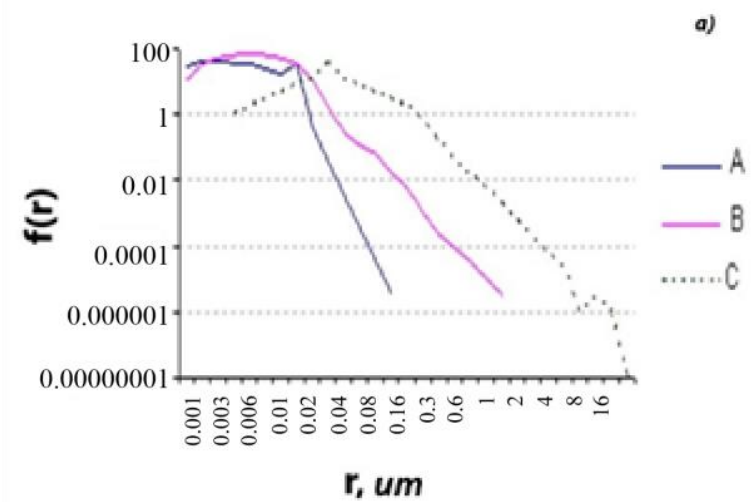

Fig. 2.

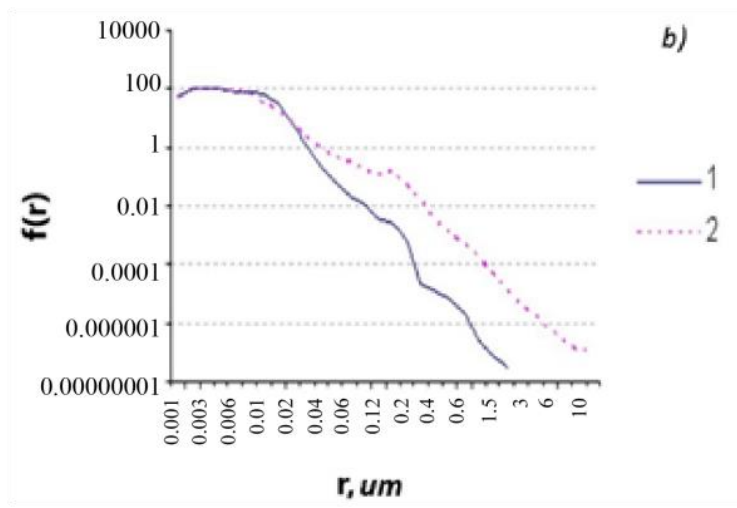

Fig. 3.

\section{References}

1. K.Ya. Kondratiev, N.I. Moskalenko, The greenhouse effect of the atmosphere VINITI (Moscow: VINITI), 260 (1984)

2. N.I. Moskalenko, Ya.S. Safiullina, M.S. Sadykova, Zonal modelling of the greenhouse effect of the atmosphere and anthropogenic climate change Alternative energy and ecology 2, 43-54 (2014)

3. N.I. Moskalenko, S.O. Mirumyants, N.F. Loktev, R. Sh. Misbakhov, Equilibrium and non-equilibrium radiation processes: high-temperature environments, radiative heat transfer ed KGEU (Kazan: KGEU), 264 (2014)

4. N.I. Moskalenko, Ya.S. Safiullina, M.S. Khamidullina, N.F. Loktev, Identification of ingredients and determination of the composition of atmospheric emissions and combustion products by 
fine-structure spectrometry Alternative energy and ecology 2, 43-54 (2010)

5. N.I. Moskalenko, Ya.S. Safiullina, Application of the method of fine-structure spectrometry to determine the ingredient composition of the products of combustion of fuels Izvestia vuzov Energy problems 11-12, 22-32 (2009)

6. N.I. Moskalenko, R.Sh. Misbakhov, I.Z. Bagautdinov, N.F. Loktev, I.R. Dodov, Determination of ingredient composition of atmospheric emissions of the turbojet engine combustion gases by the fine-structure spectroscopy Russian aeronautics 59(3), 419-425 (2016)

7. F.G. Bakirov, V.M. Zakharov, I.Z. Polishchuk, Z.G. Shaikhutdinov, Formation and burnout of soot when burning hydrocarbon fuels (Moscow: Mechanical Engineering), 128 (1989)

8. A.M. Levterov, L.I. Levterova, Analysis of mathematical models of the mechanisms of soot formation during the combustion of hydrocarbon fuels Vista NTU 5, 130-141 (2013)

9. Marig Matti, Coagulation dynamics of fractal-like soot aggregates Journal of Aerosol Science, 38(2), 141-156 (2007)

10. O.V. Vasilyeva, S.I. Ksenofontov, A.G. Krasnova, A.V. Kokshina, The structure and properties of soot in the flame of hydrocarbon fuel Alternative energy and ecology 19, 105-111 (2015)

11. V.E. Alemasov, Thermodynamic and thermophysical properties of combustion products ed VINITI (Moscow: VINITI), 490 (1972)

12. V.E. Alemasov et al, Mathematical modelling of high-temperature processes in power plants ed VINITI (Moscow: VINITI), 254 (1972)

13. N.I. Moskalenko et al, Determination of the composition of the atmospheric emissions of combustion products of a turbojet engine by the method of fine-structure spectrometry Izvestia vuzov Aviation technology 3, 116-121 (2016)

14. N.I. Moskalenko, I.R. Dodov, Fine structured spectroscopy: measuring experimental studies and their applications World chemistry forum 2019 theme: analysis, catalysis, nanoscience (Spain: Barcelona), 52 (2019)

15. L.E. Gordon et al, The HITRAN-2016 Molecular Spectroscopic Database Journal of quantitative spectroscopy and radiative transfer 203, 3-69 (2017) 